\title{
Series Solution for Steady Three-Dimensional Flow due to Spraying on Inclined Spinning Disk by Homotopy Perturbation Method
}

\author{
Saeed Dinarvand \\ Islamic Azad University, Central Tehran Branch, Young Researchers Club, Tehran, Iran \\ Correspondence should be addressed to Saeed Dinarvand, saeed_dinarvand@yahoo.com
}

Received 20 September 2011; Accepted 21 December 2011

Academic Editor: P. G. L. Leach

Copyright (C) 2012 Saeed Dinarvand. This is an open access article distributed under the Creative Commons Attribution License, which permits unrestricted use, distribution, and reproduction in any medium, provided the original work is properly cited.

The steady three-dimensional flow of condensation or spraying on inclined spinning disk is studied analytically. The governing nonlinear equations and their associated boundary conditions are transformed into the system of nonlinear ordinary differential equations. The series solution of the problem is obtained by utilizing the homotopy perturbation method (HPM). The velocity and temperature profiles are shown and the influence of Prandtl number on the heat transfer and Nusselt number is discussed in detail. The validity of our solutions is verified by the numerical results. Unlike free surface flows on an incline, this through flow is highly affected by the spray rate and the rotation of the disk.

\section{Introduction}

The removal of a condensate liquid from a cooled, saturated vapor is important in engineering processes. Sparrow and Gregg [1] considered the removal of the condensate using centrifugal forces on a cooled rotating disk. Following von Karman's [2] study of a rotating disk in an infinite fluid, Sparrow and Gregg transformed the Navier-Stokes equations into a system of nonlinear ordinary differential equations and numerically integrated for the similarity solution for several finite film thicknesses. Their work was extended by adding vapor drag by Beckett et al. [3] and adding suction on the plate by Chary and Sarma [4]. The problem is also related to chemical vapor deposition, when a thin fluid film is deposited on a cooled rotating disk [5].

Most of the scientific problems and phenomena are modeled by nonlinear ordinary or partial differential equations. In recent years, many powerful methods have been developed to construct explicit analytical solution of nonlinear differential equations. Among them, two analytical methods have drawn special attention, namely, the homotopy perturbation method (HPM) [6, 7] and homotopy analysis method (HAM) [8-11]. The essential idea in these 
methods is to introduce a homotopy parameter, say $p$, which takes the value from 0 to 1 . For $p=0$, the system of equations takes a simplified form which readily admits a particularly simple solution. When $p$ is gradually increased to 1 , the system goes through a sequence of "deformations," the solution of each of which is "close" to that at the previous stage of "deformation." Eventually at $p=1$, the system takes the original forms of equation and the final stage of "deformation" gives the desired solution.

We know all perturbation methods require small parameter in nonlinear equation and the approximate solutions of equation containing this parameter are expressed as series expansions in the small parameter. Selection of small parameter requires a special skill. A proper choice of small parameter gives acceptable results, while an improper choice may result in incorrect solutions. The homotopy perturbation method, which is a coupling of the traditional perturbation method and homotopy in topology, does not require a small parameter in equation modeling phenomena. In recent years, the HPM has been successfully employed to solve many types of linear and nonlinear problems such as the quadratic Ricatti differential equation [12], the axisymmetric flow over a stretching sheet [13], the fractional Fokker-Planck equations [14], the magnetohydrodynamic flow over a nonlinear stretching sheet [15], the thin-film flow of a fourth-grade fluid down a vertical cylinder [16], the fractional diffusion equation with absorbent term and external force [17], Burgers equation with finite transport memory [18], the system of Fredholm integral equations [19], the generalized Burger and Burger-Fisher equations [20], the wave and nonlinear diffusion equations [21], the flow through slowly expanding or contracting porous walls [22], the torsional flow of third grade fluid [23], Emden-Fowler equations [24], and Zakharov-Kuznetsov equations [25]. All of these successful applications verified the validity, effectiveness, and flexibility of the HPM.

With the above discussion in mind, the purpose of the present paper is to examine analytically the problem of condensation or spraying on an inclined rotating disk. The governing equations here are highly nonlinear coupled differential equations, which are solved by using the homotopy perturbation method. In this way, the letter has been organized as follows. In Section 2, the problem statement and mathematical formulation are presented. In Section 3, we extend the application of the HPM to construct the approximate solution for the governing equations. Section 4 contains the results and discussion. The conclusions are summarized in Section 5.

\section{Problem Statement and Mathematical Formulation}

Figure 1 shows a disk rotating in its own plane with angular velocity $\Omega$. The angle between horizontal axis and disk is $\beta$. A fluid film of thickness $t$ is formed by spraying, with the $W$ velocity. We assume the disk radius is large compared to the film thickness such that the end effects can be ignored. Vapor shear effects at the interface of vapor and fluid are usually also unimportant. The gravitational acceleration, $\bar{g}$, acts in the downward direction. The temperature on the disk is $T_{w}$ and the temperature on the film surface is $T_{0}$. Besides, the ambient pressure on the film surface is constant at $p_{0}$, and we can safely say the pressure is a function of $z$ only. Neglecting viscous dissipation, the continuity, momentum, and energy equations for steady state are given in the following form [26]:

$$
\begin{gathered}
u_{x}+v_{y}+w_{z}=0 \\
u u_{x}+v u_{y}+w u_{z}=v\left(u_{x x}+u_{y y}+u_{z z}\right)+\bar{g} \sin \beta,
\end{gathered}
$$




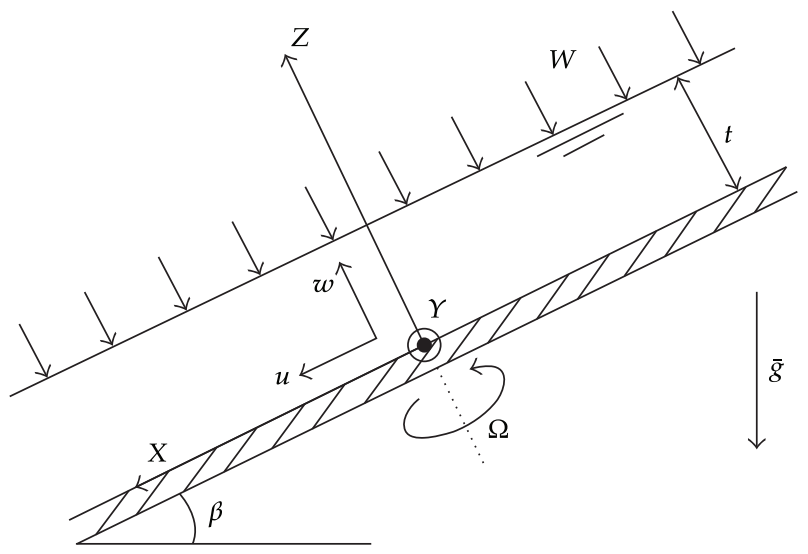

Figure 1: The schematic diagram of steady three-dimensional problem of condensation film on inclined rotating disk.

$$
\begin{gathered}
u v_{x}+v v_{y}+w v_{z}=v\left(v_{x x}+v_{y y}+v_{z z}\right), \\
u w_{x}+v w_{y}+w w_{z}=v\left(w_{x x}+w_{y y}+w_{z z}\right)-\bar{g} \cos \beta-\frac{p_{z}}{\rho}, \\
u T_{x}+v T_{y}+w T_{z}=\alpha\left(T_{x x}+T_{y y}+T_{z z}\right) .
\end{gathered}
$$

In above equations, $u, v$, and $w$ indicate the velocity components in the $x, y$, and $z$ directions, $T$ denotes the temperature, $\rho, v$, and $\alpha$ are the density, kinematic viscosity, and thermal diffusivity of the fluid, respectively. Supposing zero slip on the disk and zero shear on the film surface, the boundary conditions are

$$
\begin{gathered}
u=-\Omega y, \quad v=\Omega x, \quad w=0, \quad T=T_{w} \quad \text { at } z=0, \\
u_{z}=0, \quad v_{z}=0, \quad w=-W, \quad T=T_{0}, \quad p=p_{0} \quad \text { at } z=t .
\end{gathered}
$$

For mentioned problem, Wang introduced the following transform [26]:

$$
\begin{gathered}
u=-\Omega y g(\eta)+\Omega x f^{\prime}(\eta)+\frac{\bar{g} k(\eta) \sin \beta}{\Omega}, \\
v=\Omega x g(\eta)+\Omega y f^{\prime}(\eta)+\frac{\bar{g} s(\eta) \sin \beta}{\Omega}, \\
w=-2 \sqrt{\Omega v} f(\eta), \\
T=\left(T_{0}-T_{w}\right) \theta(\eta)+T_{w},
\end{gathered}
$$

where

$$
\eta=z \sqrt{\frac{\Omega}{v}} .
$$


Continuity equation (2.1) automatically is satisfied. Equations (2.2) and (2.3) can be written as follows:

$$
\begin{gathered}
f^{\prime \prime \prime}-\left(f^{\prime}\right)^{2}+g^{2}+2 f f^{\prime \prime}=0, \\
g^{\prime \prime}-2 g f^{\prime}+2 f g^{\prime}=0, \\
k^{\prime \prime}-k f^{\prime}+s g+2 f k^{\prime}+1=0, \\
s^{\prime \prime}-g k-s f^{\prime}+2 f s^{\prime}=0 .
\end{gathered}
$$

If the temperature is a function of the distance $z$ only, (2.5) becomes

$$
\theta^{\prime \prime}+2 \operatorname{Pr} f \theta^{\prime}=0
$$

where $\operatorname{Pr}=v / \alpha$ is the Prandtl number. The boundary conditions for (2.9)-(2.10) are

$$
\begin{array}{cl}
f(0)=0, \quad f^{\prime}(0)=0, \quad f^{\prime \prime}(\delta)=0, \\
g(0)=1, \quad g^{\prime}(\delta)=0, \\
k(0)=0, \quad k^{\prime}(\delta)=0, \\
s(0)=0, \quad s^{\prime}(\delta)=0, \\
\theta(0)=0, \quad \theta(\delta)=1,
\end{array}
$$

and $\delta$ is the constant normalized thickness as

$$
\delta=t \sqrt{\frac{\Omega}{v}}
$$

After the flow field is found, the pressure can be obtained by integrating (2.4):

$$
p(z)=p_{0}+\rho\left\{v\left[w_{z}(z)-w_{z}(t)\right]-\frac{\left[w^{2}(z)-w^{2}(t)\right]}{2}-\bar{g}(z-t) \cos \beta\right\} .
$$

\section{Solution by Homotopy Perturbation Method}

\subsection{Basic Idea}

Now, for convenience, consider the following general nonlinear differential equation:

$$
A(u)-f(r)=0, \quad r \in \Omega,
$$


with boundary conditions:

$$
B\left(u, \frac{\partial u}{\partial n}\right)=0, \quad r \in \Gamma
$$

where $A$ is a general differential operator, $B$ is a boundary operator, $f(r)$ is a known analytic function, and $\Gamma$ is the boundary of the domain $\Omega$.

The operator $A$ can, generally speaking, be divided into two parts $L$ and $N$, where $L$ is linear, and $N$ is nonlinear, therefore, (3.1) can be written as

$$
L(u)+N(u)-f(r)=0 .
$$

By using homotopy technique, one can construct a homotopy $\tilde{u}(r, p): \Omega \times[0,1] \rightarrow R$ which satisfies

$$
H(\tilde{u}, p)=(1-p)\left[L(\tilde{u})-L\left(u_{0}\right)\right]+p[A(\tilde{u})-f(r)]=0,
$$

or

$$
H(\tilde{u}, p)=L(\widetilde{u})-L\left(u_{0}\right)+p L\left(u_{0}\right)+p[N(\widetilde{u})-f(r)]=0,
$$

where $p \in[0,1]$ is an embedding parameter, and $u_{0}$ is the initial approximation of (3.1) which satisfies the boundary conditions. Clearly, we have

$$
\begin{gathered}
H(\tilde{u}, 0)=L(\tilde{u})-L\left(u_{0}\right)=0, \\
H(\tilde{u}, 1)=A(\tilde{u})-f(r)=0 .
\end{gathered}
$$

The changing process of $p$ from zero to unity is just that of $\tilde{u}(r, p)$ changing from $u_{0}(r)$ to $u(r)$. This is called deformation, and also $L(\tilde{u})-L\left(u_{0}\right)$ and $A(\tilde{u})-f(r)$ are called homotopic in topology. If the embedding parameter $p(0 \leq p \leq 1)$ is considered as a "small parameter," applying the classical perturbation technique, we can naturally assume that the solution of (3.4) and (3.5) can be given as a power series in $p$ :

$$
\tilde{u}=\tilde{u}_{0}+p \tilde{u}_{1}+p^{2} \tilde{u}_{2}+\cdots
$$

and setting $p=1$ results in the approximate solution of (3.1) as

$$
u=\lim _{p \rightarrow 1} \tilde{u}=\tilde{u}_{0}+\tilde{u}_{1}+\tilde{u}_{2}+\cdots .
$$

The convergence of series (3.8) has been proved by He in his paper [27]. It is worth to note that the major advantage of He homotopy perturbation method is that the perturbation equation can be freely constructed in many ways (therefore, it is problem dependent) by homotopy in topology, and the initial approximation can also be freely selected. Moreover, the construction of the homotopy for the perturb problem plays very important role for obtaining desired accuracy. 


\subsection{Guidelines for Choosing Homotopy Equation}

In a homotopy equation, what we are mainly concerned about are the auxiliary linear operator $L$ and the initial approximation $u_{0}$. Once one chooses these parts, the homotopy equation is completely determined, because the remaining part is actually the original equation (3.5) and we have less freedom to change it. Here, we discuss some general rules that should be noted in choosing $L$ and $u_{0}$.

\subsubsection{Discussion on Auxiliary Linear Operator L}

According to the steps of the homotopy perturbation method, $L$ should be as follows.

\section{(i) Easy to Handle}

We mean that it must be chosen in such a way that one has no difficulty in subsequently solving systems of resulting equations. It should be noted that this condition does not restrict $L$ to be linear. In scarce cases, as was done by He in [27] to solve the Lighthill equation, a nonlinear choice of $L$ may be more suitable; But it is strongly recommended for beginners to take a linear operator as $L$.

\section{(ii) Closely Related to the Original Equation}

Strictly speaking, in constructing $L$, it is better to use some part of the original equation [23]. We can see the effectiveness of this view in [28] where Chowdhury and Hashim have gained very good results with technically choosing the $L$ part.

\subsubsection{Discussion on Initial Approximation $u_{0}$}

There is no unique universal technique for choosing the initial approximation in iterative methods, but from previous works done on HPM $[29,30]$ and our own experiences, we can conclude the following facts.

\section{(i) It Should Be Obtained from the Original Equation}

For example, it can be chosen to be the solution to some part of the original equation, or it can be chosen from initial/boundary conditions.

\section{(ii) It Should Reduce Complexity of the Resulting Equations}

Although this condition only can be checked after solving some of the first few equations of the resulting system, this is the criteria that has been used by many authors when they encountered different choices as an initial approximation.

\subsubsection{Completive Discussion on Auxiliary Convergence-Control Parameter}

An important point to note is that, whenever we apply an auxiliary parameter as a factor for second term of homotopy equation (3.4), we have an option for convergence control of series 
solution. Foregoing parameter would be applicable similar to convergence-control parameter in homotopy analysis method pointed out by Liao [31].

\subsection{Application for Steady Three-Dimensional Flow of Condensation Film on Inclined Spinning Disk}

To investigate the explicit and totally analytic solutions of present problem by using HPM, we first define homotopy $\tilde{f}(\eta, p): \Omega \times[0,1] \rightarrow \mathcal{R}, \tilde{g}(\eta, p): \Omega \times[0,1] \rightarrow \mathcal{R}, \tilde{k}(\eta, p): \Omega \times[0,1] \rightarrow \mathcal{R}$, $\widetilde{\mathrm{s}}(\eta, p): \Omega \times[0,1] \rightarrow \mathcal{R}$, and $\tilde{\theta}(\eta, p): \Omega \times[0,1] \rightarrow \mathcal{R}$ for (2.9)-(2.10) which satisfies

$$
\begin{gathered}
(1-p)\left[L_{1}(\tilde{f})-L_{1}\left(f_{0}\right)\right]+p\left[\tilde{f}^{\prime \prime \prime}-\left(\tilde{f}^{\prime}\right)^{2}+\tilde{g}^{2}+2 \tilde{f} \tilde{f}^{\prime \prime}\right]=0 \\
(1-p)\left[L_{2}(\tilde{g})-L_{2}\left(g_{0}\right)\right]+p\left[\tilde{g}^{\prime \prime}-2 \tilde{g} \tilde{f}^{\prime}+2 \tilde{f} \tilde{g}^{\prime}\right]=0 \\
(1-p)\left[L_{3}(\tilde{k})-L_{3}\left(k_{0}\right)\right]+p\left[\tilde{k}{ }^{\prime \prime}-\tilde{k} \tilde{f}^{\prime}+\tilde{s} \tilde{g}+2 \tilde{f} \tilde{k}^{\prime}+1\right]=0 \\
(1-p)\left[L_{4}(\tilde{s})-L_{4}\left(s_{0}\right)\right]+p\left[\tilde{s}^{\prime \prime}-\tilde{g} \tilde{k}-\tilde{s} \tilde{f}^{\prime}+2 \tilde{f} \widetilde{s}^{\prime}\right]=0 \\
(1-p)\left[L_{5}(\tilde{\theta})-L_{5}\left(\theta_{0}\right)\right]+p\left[\tilde{\theta}^{\prime \prime}+2 \operatorname{Pr} \tilde{f} \tilde{\theta}^{\prime}\right]=0
\end{gathered}
$$

where $L_{1}, L_{2}, L_{3}, L_{4}$, and $L_{5}$ are linear operators as follows:

$$
\begin{aligned}
& L_{1}(\tilde{f})=\frac{d^{3} \tilde{f}}{d \eta^{3}} \\
& L_{2}(\tilde{g})=\frac{d^{2} \tilde{g}}{d \eta^{2}} \\
& L_{3}(\widetilde{k})=\frac{d^{2} \tilde{k}}{d \eta^{2}} \\
& L_{4}(\widetilde{s})=\frac{d^{2} \widetilde{s}}{d \eta^{2}}, \\
& L_{5}(\tilde{\theta})=\frac{d^{2} \tilde{\theta}}{d \eta^{2}} .
\end{aligned}
$$

We choose

$$
\begin{gathered}
f_{0}(\eta)=\frac{1}{3}\left(3 \delta \eta^{2}-\eta^{3}\right), \\
g_{0}(\eta)=1
\end{gathered}
$$




$$
\begin{gathered}
k_{0}(\eta)=\frac{1}{2}\left(2 \delta \eta-\eta^{2}\right), \\
s_{0}(\eta)=\frac{1}{2}\left(2 \delta \eta-\eta^{2}\right), \\
\theta_{0}(\eta)=\frac{\eta}{\delta}
\end{gathered}
$$

as initial approximations of $f(\eta), g(\eta), k(\eta), s(\eta)$, and $\theta(\eta)$, which satisfy the boundary conditions (2.11). Assume the solutions of (3.9) have the forms:

$$
\begin{aligned}
& \tilde{f}(\eta)=\tilde{f}_{0}(\eta)+p \tilde{f}_{1}(\eta)+p^{2} \tilde{f}_{2}(\eta)+\cdots, \\
& \tilde{g}(\eta)=\tilde{g}_{0}(\eta)+p \tilde{g}_{1}(\eta)+p^{2} \tilde{g}_{2}(\eta)+\cdots, \\
& \tilde{k}(\eta)=\tilde{k}_{0}(\eta)+p \tilde{k}_{1}(\eta)+p^{2} \tilde{k}_{2}(\eta)+\cdots, \\
& \tilde{s}(\eta)=\widetilde{s}_{0}(\eta)+p \widetilde{s}_{1}(\eta)+p^{2} \widetilde{s}_{2}(\eta)+\cdots, \\
& \tilde{\theta}(\eta)=\tilde{\theta}_{0}(\eta)+p \tilde{\theta}_{1}(\eta)+p^{2} \tilde{\theta}_{2}(\eta)+\cdots,
\end{aligned}
$$

where $\tilde{f}_{i}(\eta), \tilde{g}_{i}(\eta), \tilde{k}_{i}(\eta), \tilde{s}_{i}(\eta)$, and $\tilde{\theta}_{i}(\eta), i=1,2,3, \ldots$ are functions yet to be determined. Substituting (3.12) into (3.9) and equating the terms with identical powers of $p$, we have

$$
p^{0} \Longrightarrow\left\{\begin{array}{l}
\tilde{f}_{0}^{\prime \prime \prime}-f_{0}^{\prime \prime \prime}=0, \\
\tilde{f}_{0}(0)=0, \tilde{f}_{0}^{\prime}(0)=0, \tilde{f}_{0}^{\prime \prime}(\delta)=0, \\
\tilde{g}_{0}^{\prime \prime}-g_{0}^{\prime \prime}=0, \\
\tilde{g}_{0}(0)=1, \tilde{g}_{0}^{\prime}(\delta)=0, \\
\tilde{k}_{0}^{\prime \prime}-k_{0}^{\prime \prime}=0, \\
\tilde{k}_{0}(0)=0, \tilde{k}_{0}^{\prime}(\delta)=0, \\
\tilde{s}_{0}^{\prime \prime}-s_{0}^{\prime \prime}=0, \\
\tilde{s}_{0}(0)=0, \widetilde{s}_{0}^{\prime}(\delta)=0, \\
\tilde{\theta}_{0}^{\prime \prime}-\theta_{0}^{\prime \prime}=0, \\
\tilde{\theta}_{0}(0)=0, \tilde{\theta}_{0}(\delta)=1,
\end{array}\right.
$$


Journal of Applied Mathematics

$$
\begin{aligned}
& p^{1} \Longrightarrow\left\{\begin{array}{l}
\tilde{f}_{1}^{\prime \prime \prime}+f_{0}^{\prime \prime \prime}-\tilde{f}_{0}^{\prime 2}+\tilde{g}_{0}^{2}+2 \tilde{f}_{0} \tilde{f}_{0}^{\prime \prime}=0 \\
\tilde{f}_{1}(0)=0, \tilde{f}_{1}^{\prime}(0)=0, \tilde{f}_{1}^{\prime \prime}(\delta)=0 \\
\tilde{g}_{1}^{\prime \prime}+g_{0}^{\prime \prime}-2 \tilde{g}_{0} \tilde{f}_{0}^{\prime}+2 \tilde{f}_{0} \tilde{g}_{0}^{\prime}=0 \\
\tilde{g}_{1}(0)=0, \tilde{g}_{1}^{\prime}(\delta)=0 \\
\tilde{k}_{1}^{\prime \prime}+k_{0}^{\prime \prime}-\tilde{k}_{0} \tilde{f}_{0}^{\prime}+\tilde{s}_{0} \tilde{g}_{0}+2 \tilde{f}_{0} \tilde{k}_{0}^{\prime}+1=0, \\
\tilde{k}_{1}(0)=0, \tilde{k}_{1}^{\prime}(\delta)=0 \\
\tilde{s}_{1}^{\prime \prime}+s_{0}^{\prime \prime}-\tilde{k}_{0} \tilde{g}_{0}-\widetilde{s}_{0} \tilde{f}_{0}^{\prime}+2 \tilde{f}_{0} \tilde{s}_{0}^{\prime}=0 \\
\tilde{s}_{1}(0)=0, \tilde{s}_{1}^{\prime}(\delta)=0 \\
\tilde{\theta}_{1}^{\prime \prime}+\theta_{0}^{\prime \prime}+2 \operatorname{Pr} \tilde{f}_{0} \tilde{\theta}_{0}^{\prime}=0 \\
\tilde{\theta}_{1}(0)=0, \tilde{\theta}_{1}(\delta)=0
\end{array}\right. \\
& p^{2} \Longrightarrow\left\{\begin{array}{l}
\tilde{f}_{2}^{\prime \prime \prime}-2 \tilde{f}_{0}^{\prime} \tilde{f}_{1}^{\prime}+2 \tilde{g}_{0} \tilde{g}_{1}+2 \tilde{f}_{0} \tilde{f}_{1}^{\prime \prime}+2 \tilde{f}_{1} \tilde{f}_{0}^{\prime \prime}=0, \\
\tilde{f}_{2}(0)=0, \tilde{f}_{2}^{\prime}(0)=0, \tilde{f}_{2}^{\prime \prime}(\delta)=0, \\
\tilde{g}_{0}^{\prime \prime}-2 \widetilde{g}_{0} \tilde{f}_{1}^{\prime}-2 \tilde{g}_{1} \tilde{f}_{0}^{\prime}+2 \tilde{f}_{0} \tilde{g}_{1}^{\prime}+2 \tilde{f}_{1} \tilde{g}_{0}^{\prime}=0, \\
\tilde{g}_{2}(0)=0, \tilde{g}_{2}^{\prime}(\delta)=0, \\
\tilde{k}_{2}^{\prime \prime}-\tilde{k}_{0} \tilde{f}_{1}^{\prime}-\tilde{k}_{1} \tilde{f}_{0}^{\prime}+\widetilde{s}_{0} \tilde{g}_{1}+\tilde{s}_{1} \tilde{g}_{0}+2 \tilde{f}_{0} \tilde{k}_{1}^{\prime}+2 \tilde{f}_{1} \tilde{k}_{0}^{\prime}=0, \\
\tilde{k}_{2}(0)=0, \tilde{k}_{2}^{\prime}(\delta)=0, \\
\tilde{s}_{2}^{\prime \prime}-\tilde{k}_{0} \tilde{g}_{1}-\tilde{k}_{1} \tilde{g}_{0}-\widetilde{s}_{0} \tilde{f}_{1}^{\prime}-\widetilde{s}_{1} \tilde{f}_{0}^{\prime}+2 \tilde{f}_{0} \tilde{s}_{1}^{\prime}+2 \tilde{f}_{1} \tilde{s}_{0}^{\prime}=0, \\
\tilde{s}_{2}(0)=0, \tilde{s}_{2}^{\prime}(\delta)=0, \\
\tilde{\theta}_{2}^{\prime \prime}+2 \operatorname{Pr} \tilde{f}_{0} \tilde{\theta}_{1}^{\prime}+2 \operatorname{Pr} \tilde{f}_{1} \tilde{\theta}_{0}^{\prime \prime}=0, \\
\tilde{\theta}_{2}(0)=0, \tilde{\theta}_{2}(\delta)=0,
\end{array}\right.
\end{aligned}
$$

The solutions of system (3.13) may be written as follows:

$$
\begin{gathered}
\tilde{f}_{0}(\eta)=\frac{1}{3}\left(3 \delta \eta^{2}-\eta^{3}\right), \\
\tilde{g}_{0}(\eta)=1, \\
\tilde{k}_{0}(\eta)=\frac{1}{2}\left(2 \delta \eta-\eta^{2}\right),
\end{gathered}
$$




$$
\begin{gathered}
\tilde{s}_{0}(\eta)=\frac{1}{2}\left(2 \delta \eta-\eta^{2}\right) \\
\tilde{\theta}_{0}(\eta)=\frac{\eta}{\delta} \\
\tilde{f}_{1}(\eta)=-\frac{1}{630}\left[\left(315 \delta+84 \delta^{5}\right) \eta^{2}-105 \eta^{3}-7 \delta \eta^{6}+\eta^{7}\right] \\
\tilde{g}_{1}(\eta)=-\frac{1}{6}\left[8 \delta^{3} \eta-4 \delta \eta^{3}+\eta^{4}\right] \\
\tilde{k}_{1}(\eta)=-\frac{1}{360}\left[\left(-120 \delta^{3}+48 \delta^{5}\right) \eta+60 \delta \eta^{3}-15 \eta^{4}-12 \delta \eta^{5}+2 \eta^{6}\right] \\
\tilde{s}_{1}(\eta)=-\frac{1}{360}\left[\left(360 \delta+120 \delta^{3}+48 \delta^{5}\right) \eta-180 \eta^{2}-60 \delta \eta^{3}+15 \eta^{4}-12 \delta \eta^{5}+2 \eta^{6}\right] \\
\tilde{\theta}_{1}(\eta)=-\frac{\operatorname{Pr}}{30 \delta}\left[-4 \delta^{4} \eta+5 \delta \eta^{4}-\eta^{5}\right]
\end{gathered}
$$

In the same manner, the rest of components were obtained using the MATHEMATICA Package. According to the HPM, we can conclude that

$$
\begin{aligned}
& f(\eta)=\lim _{p \rightarrow 1} \tilde{f}(\eta)=\tilde{f}_{0}(\eta)+\tilde{f}_{1}(\eta)+\tilde{f}_{2}(\eta)+\cdots, \\
& g(\eta)=\lim _{p \rightarrow 1} \tilde{g}(\eta)=\tilde{g}_{0}(\eta)+\tilde{g}_{1}(\eta)+\tilde{g}_{2}(\eta)+\cdots, \\
& k(\eta)=\lim _{p \rightarrow 1} \tilde{k}(\eta)=\tilde{k}_{0}(\eta)+\tilde{k}_{1}(\eta)+\tilde{k}_{2}(\eta)+\cdots, \\
& s(\eta)=\lim _{p \rightarrow 1} \tilde{s}(\eta)=\tilde{s}_{0}(\eta)+\widetilde{s}_{1}(\eta)+\widetilde{s}_{2}(\eta)+\cdots, \\
& \theta(\eta)=\lim _{p \rightarrow 1} \tilde{\theta}(\eta)=\tilde{\theta}_{0}(\eta)+\tilde{\theta}_{1}(\eta)+\tilde{\theta}_{2}(\eta)+\cdots,
\end{aligned}
$$

\section{Results and Discussion}

Figures 2 and 3 show the normalized velocity profiles $f(\eta), f^{\prime}(\eta), g(\eta), k(\eta)$, and $s(\eta)$ obtained by the HPM in comparison with the numerical solution by the fourth-order RungeKutta method. From Figures 2 and 3, we can see a very good agreement between the purely analytic results of the HPM and numerical results. The net flow due to the tilting of the disk includes two components. The net draining flow per width in the $x$ direction and induced net flow in the lateral $y$ direction, normalized by $\bar{g} \sqrt{v / \Omega^{3}} \sin \beta$, are $\int_{0}^{\delta} k(\eta) d \eta$ and $\int_{0}^{\delta} s(\eta) d \eta$, respectively. Figure 4 shows the normalized draining and lateral flow rate obtained by the HPM. Another one of the important quantities is the net force on the disk. 


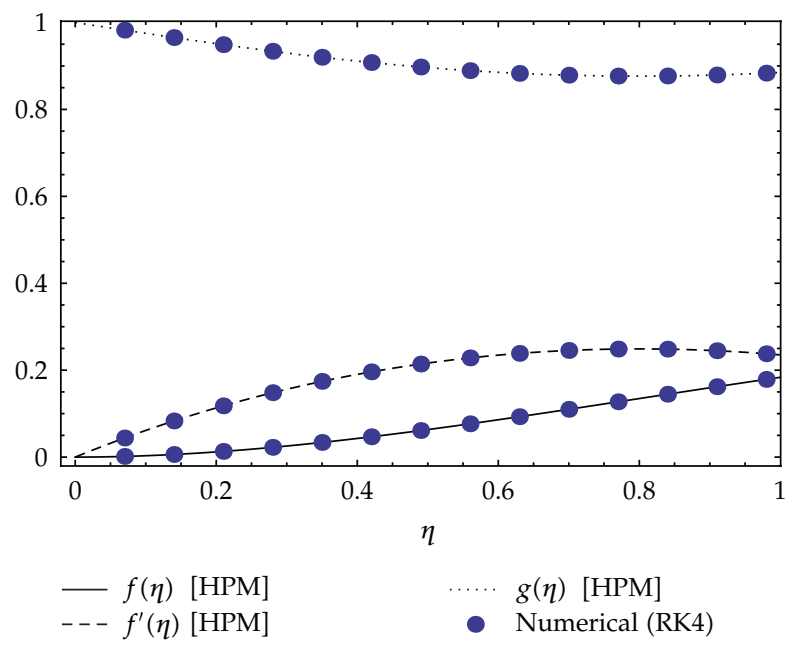

Figure 2: The normalized radial velocity profiles for the rotating flow obtained by the 8th-order approximation of the HPM in comparison with the numerical solution, when $\delta=0.8$.

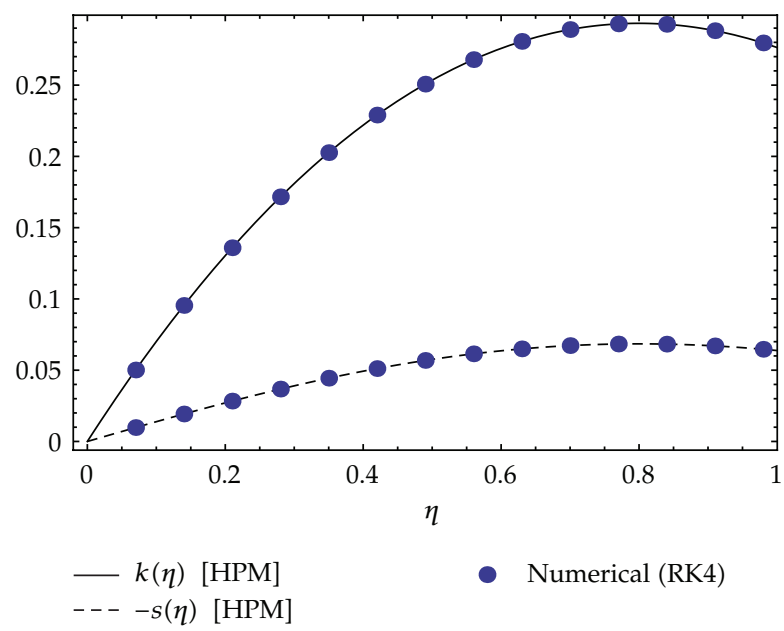

Figure 3: The normalized velocity profiles for the draining flow $(k(\eta))$ and lateral flow $(s(\eta))$ obtained by the 8th-order approximation of the HPM in comparison with the numerical solution, when $\delta=0.8$.

The net forces per unit area in the $x$ and $y$ directions, normalized by $\rho \bar{g} \sqrt{v / \Omega} \sin \beta$, are $k^{\prime}(0)$ and $s^{\prime}(0)$, respectively. Figure 5 shows these quantities. The normalized temperature profiles for different values of the Prandtl number are represented in Figure 6. The value of $\theta^{\prime}(0)$ represents the local heat transfer on the disk normalized by $\bar{k}\left(T_{0}-T_{w}\right) \sqrt{\Omega / v}$, where $\bar{k}$ is the thermal conductivity. We show $\theta^{\prime}(0)$ for different values of the Prandtl number in Figure 7. The Nusselt number is defined by

$$
\mathrm{Nu}=\frac{(\partial T / \partial z)_{w}}{T_{0}-T_{w}}=\delta \theta^{\prime}(0)
$$




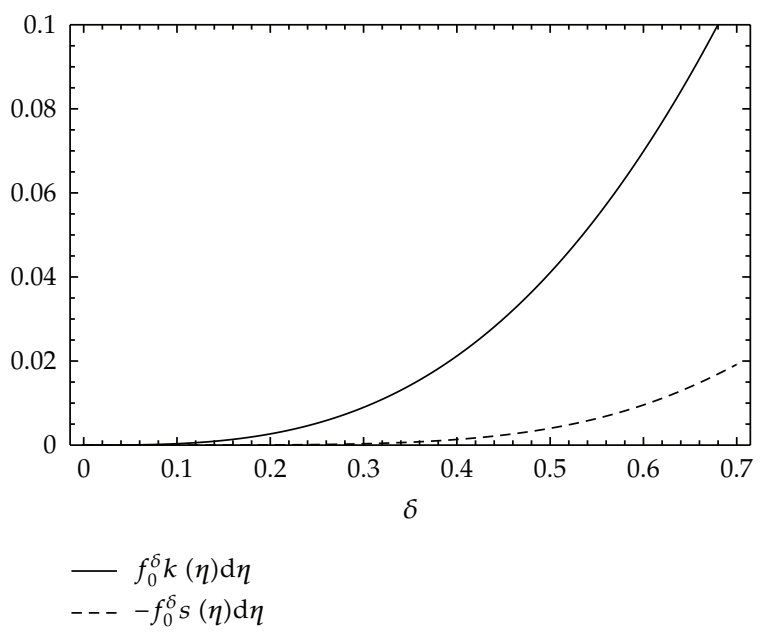

Figure 4: The normalized draining flow rate $\left(\int_{0}^{\delta} k(\eta) d \eta\right)$ and the normalized lateral flow rate $\left(\int_{0}^{\delta} s(\eta) d \eta\right)$ obtained by the 8th-order approximation of the HPM.

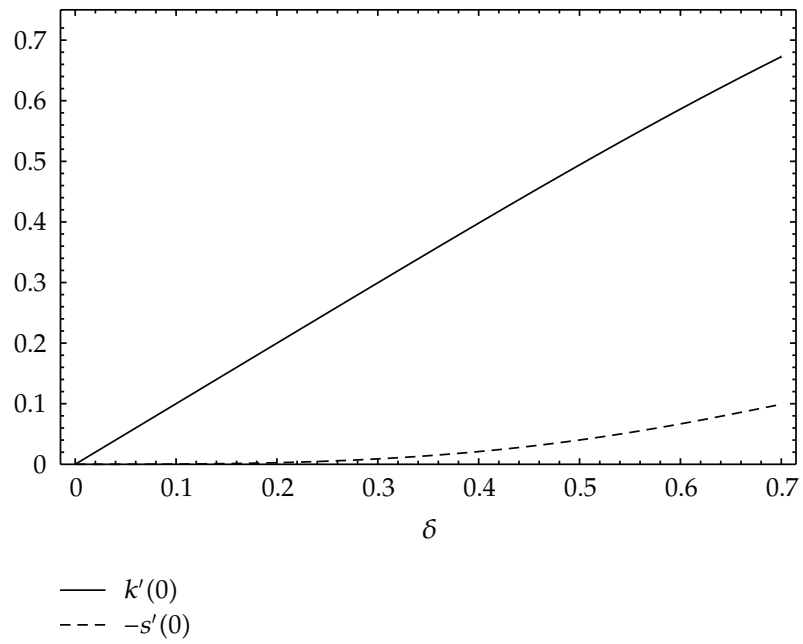

Figure 5: The normalized shear on the disk in the $x\left(k^{\prime}(0)\right)$ and $y\left(s^{\prime}(0)\right)$ directions, obtained by the 8th-order approximation of the HPM.

The Nusselt number for different values of the Prandtl number is illustrated in Figure 8. This figure elucidates that the Nusselt number increases with thickness. Furthermore, the Nusselt number approaches one as thickness tends to zero.

\section{Final Remarks}

In this paper, the homotopy perturbation method (HPM) was used for finding the totally analytic solutions of the system of nonlinear ordinary differential equations derived from similarity transform for the steady three-dimensional problem of fluid deposition on an inclined rotating disk. The analytical results depicted by the graphs are consistent with 


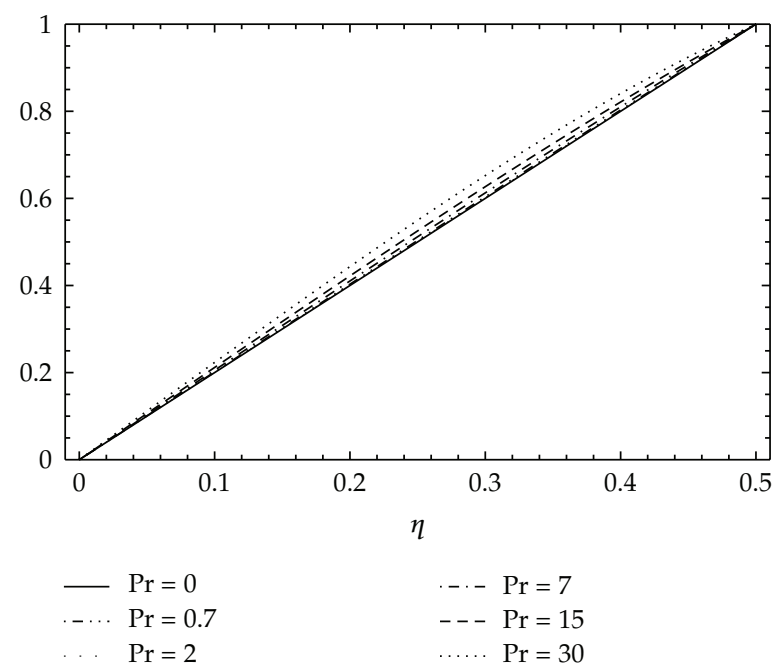

Figure 6: The normalized temperature profiles $(\theta(\eta))$ obtained by the 8th-order approximation of the HPM for different values of the Prandtl number, when $\delta=0.5$.

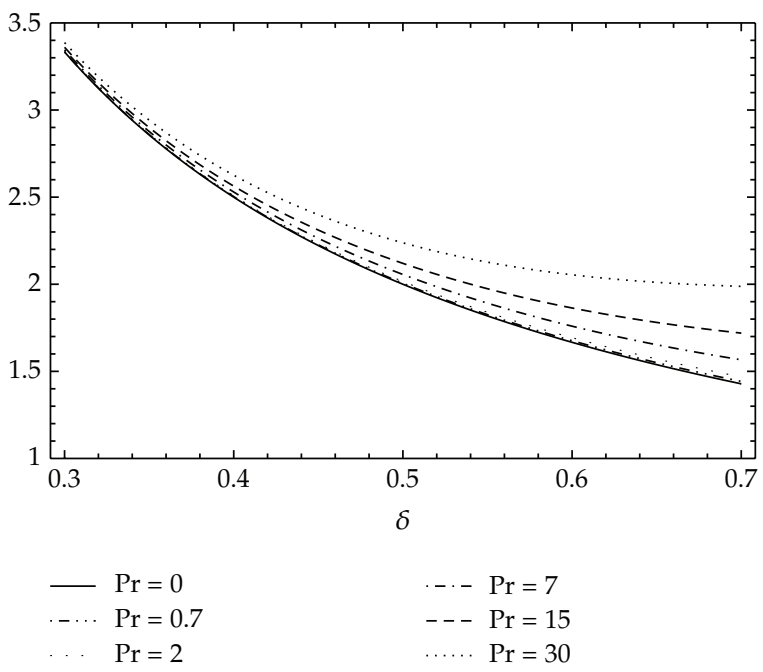

Figure 7: The temperature gradient on the disk $\left(\theta^{\prime}(0)\right)$ obtained by the 8th-order approximation of the HPM for different values of the Prandtl number.

the graphs produced by the fourth-order Runge-Kutta method, and, therefore, further establish the reliability and effectiveness of the HPM solution. This method provides an analytical approximate solution without any assumption of linearization. This character is very important for systems with strong nonlinearities which could be extremely sensitive to small changes in parameters. The solution obtained by means of HPM is an infinite power series for appropriate initial approximation, which can be, in turn, expressed in a closed form. In this regard, the homotopy perturbation method is found to be a very useful analytic technique to get highly accurate and purely analytic solution to such kind of nonlinear problems. 


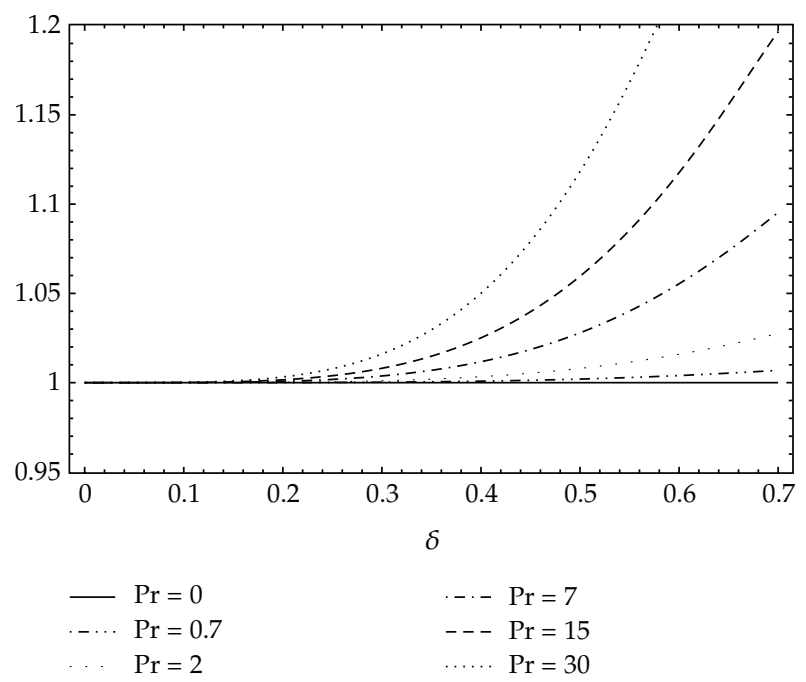

Figure 8: The Nusselt number $\left(\delta \theta^{\prime}(0)\right)$ obtained by the 8th-order approximation of the HPM for different values of the Prandtl number.

\section{References}

[1] E. M. Sparrow and J. L. Gregg, "A theory of rotating condensation," Journal of Heat Transfer, vol. 81, pp. 113-120, 1959.

[2] T. von Karman, "Uber laminare und turbulente reibung," Zeitschrift für Angewandte Mathematik und Mechanik, vol. 1, no. 4, pp. 233-252, 1921.

[3] P. M. Beckett, P. C. Hudson, and G. Poots, "Laminar film condensation due to a rotating disk," Journal of Engineering Mathematics, vol. 7, no. 1, pp. 63-73, 1973.

[4] S. P. Chary and P. K. Sarma, "Condensation on a rotating disk with constant axial suction," Journal of Heat Transfer, vol. 98, no. 4, pp. 682-684, 1976.

[5] K. F. Jensen, E. O. Einset, and D. I. Fotiadis, "Flow phenomena in chemical vapor deposition of thin films," Annual Review of Fluid Mechanics, vol. 23, no. 1, pp. 197-232, 1991.

[6] J.-H. He, "A coupling method of a homotopy technique and a perturbation technique for non-linear problems," International Journal of Non-Linear Mechanics, vol. 35, no. 1, pp. 37-43, 2000.

[7] J.-H. He, "Homotopy perturbation method for solving boundary value problems," Physics Letters A, vol. 350, no. 1-2, pp. 87-88, 2006.

[8] Liao, The proposed homotopy analysis technique for the solution of non-linear problems, Ph.D. thesis, Shanghai Jiao Tong University, Shanghai, China, 1992.

[9] S. Dinarvand, "A reliable treatment of the homotopy analysis method for viscous flow over a nonlinearly stretching sheet in presence of a chemical reaction and under influence of a magnetic field," Central European Journal of Physics, vol. 7, no. 1, pp. 114-122, 2009.

[10] S. Dinarvand, "On explicit, purely analytic solutions of off-centered stagnation flow towards a rotating disc by means of HAM," Nonlinear Analysis. Real World Applications, vol. 11, no. 5, pp. 33893398, 2010.

[11] S. Dinarvand, "The laminar free-convection boundary-layer flow about a heated and rotating downpointing vertical cone in the presence of a transverse magnetic field," International Journal for Numerical Methods in Fluids, vol. 67, no. 12, pp. 2141-2156, 2011.

[12] S. Abbasbandy, "Iterated He's homotopy perturbation method for quadratic Riccati differential equation," Applied Mathematics and Computation, vol. 175, no. 1, pp. 581-589, 2006.

[13] P. D. Ariel, T. Hayat, and S. Asghar, "Homotopy perturbation method and axisymmetric flow over a stretching sheet," International Journal of Nonlinear Sciences and Numerical Simulation, vol. 7, no. 4, pp. 399-406, 2006.

[14] M. M. Mousa and A. Kaltayev, "Application of He's homotopy perturbation method for solving fractional Fokker-Planck equations," Zeitschrift fur Naturforschung, vol. 64, no. 12, pp. 788-794, 2009. 
[15] A. Yildirim and S. A. Sezer, "Non-perturbative solution of the magnetohydrodynamic flow over a nonlinear stretching sheet by homotopy perturbation method-pad technique," Zeitschrift fur Naturforschung, vol. 65, no. 12, pp. 1106-1110, 2010.

[16] A. M. Siddiqui, R. Mahmood, and Q. K. Ghori, "Homotopy perturbation method for thin film flow of a fourth grade fluid down a vertical cylinder," Physics Letters A, vol. 352, no. 4-5, pp. 404-410, 2006.

[17] S. Das and P. K. Gupta, "An approximate analytical solution of the fractional diffusion equation with absorbent term and external force by homotopy perturbation method," Zeitschrift fur Naturforschung, vol. 65, no. 3, pp. 182-190, 2010.

[18] R. Sakthivel, C. Chun, and J. Lee, "New travelling wave solutions of burgers equation with finite transport memory," Zeitschrift fur Naturforschung, vol. 65, no. 8, pp. 633-640, 2010.

[19] M. Javidi and A. Golbabai, "A numerical solution for solving system of Fredholm integral equations by using homotopy perturbation method," Applied Mathematics and Computation, vol. 189, no. 2, pp. 1921-1928, 2007.

[20] M. M. Rashidi, D. D. Ganji, and S. Dinarvand, "Explicit analytical solutions of the generalized Burger and Burger-Fisher equations by homotopy perturbation method," Numerical Methods for Partial Differential Equations, vol. 25, no. 2, pp. 409-417, 2009.

[21] C. Chun, H. Jafari, and Y.-I. Kim, "Numerical method for the wave and nonlinear diffusion equations with the homotopy perturbation method," Computers \& Mathematics with Applications, vol. 57, no. 7, pp. 1226-1231, 2009.

[22] S. Dinarvand, "Viscous flow through slowly expanding or contracting porous walls with low seepage Reynolds number: a model for transport of biological fluids through vessels," Computer Methods in Biomechanics and Biomedical Engineering, vol. 14, no. 10, pp. 853-862, 2011.

[23] A. M. Siddiqui, T. Haroon, and S. Irum, "Torsional flow of third grade fluid using modified homotopy perturbation method," Computers \& Mathematics with Applications, vol. 58, no. 11-12, pp. 2274-2285, 2009.

[24] M. S. H. Chowdhury and I. Hashim, "Solutions of Emden-Fowler equations by homotopyperturbation method," Nonlinear Analysis. Real World Applications, vol. 10, no. 1, pp. 104-115, 2009.

[25] J. Biazar, F. Badpeima, and F. Azimi, "Application of the homotopy perturbation method to ZakharovKuznetsov equations," Computers $\mathcal{E}$ Mathematics with Applications, vol. 58, no. 11-12, pp. 2391-2394, 2009.

[26] C. Y. Wang, "Condensation film on an inclined rotating disk," Applied Mathematical Modelling, vol. 31, no. 8, pp. 1582-1593, 2007.

[27] J.-H. He, "Homotopy perturbation technique," Computer Methods in Applied Mechanics and Engineering, vol. 178, no. 3-4, pp. 257-262, 1999.

[28] M. S. H. Chowdhury and I. Hashim, "Solutions of time-dependent Emden-Fowler type equations by homotopy-perturbation method," Physics Letters A, vol. 368, no. 3-4, pp. 305-313, 2007.

[29] E. Hesameddini and H. Latifizadeh, "An optimal choice of initial solutions in the homotopy perturbation method," International Journal of Nonlinear Sciences and Numerical Simulation, vol. 10, no. 11-12, pp. 1389-1398, 2009.

[30] E. Hesameddini and H. Latifizadeh, "A new vision of the He's homotopy perturbation method," International Journal of Nonlinear Sciences and Numerical Simulation, vol. 10, no. 11-12, pp. 1415-1424, 2009.

[31] S. J. Liao, Beyond Perturbation: Introduction to the Homotopy Analysis Method, Chapman \& Hall Press, Boca Raton, Fla, USA, 2004. 


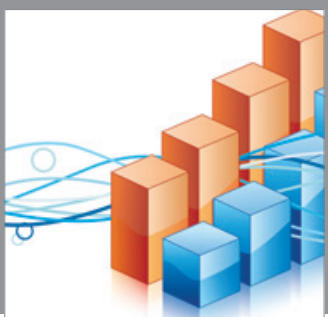

Advances in

Operations Research

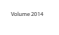

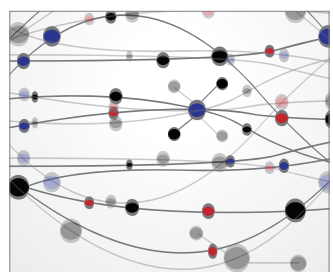

\section{The Scientific} World Journal
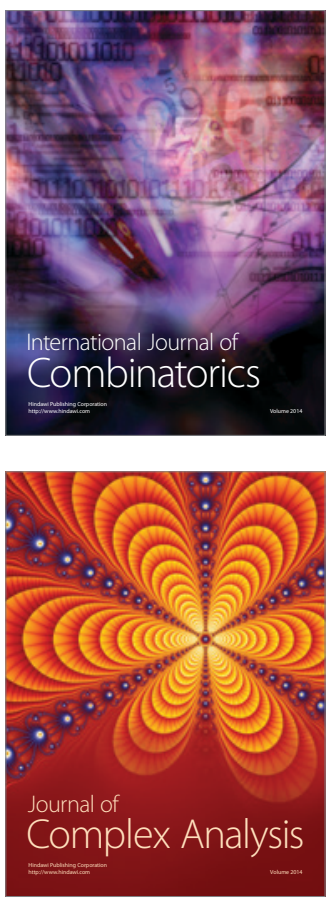

International Journal of

Mathematics and

Mathematical

Sciences
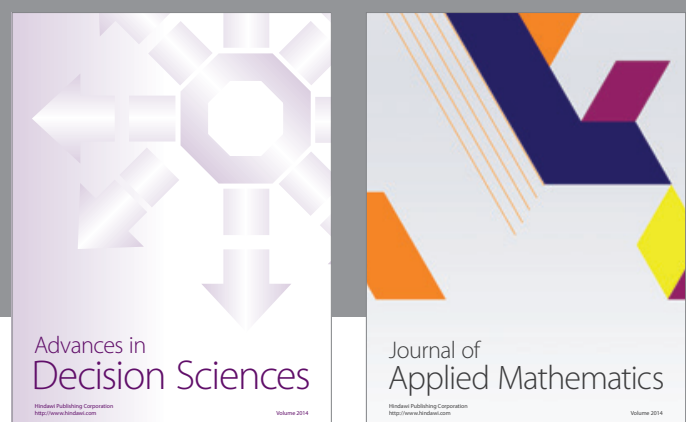

Journal of

Applied Mathematics
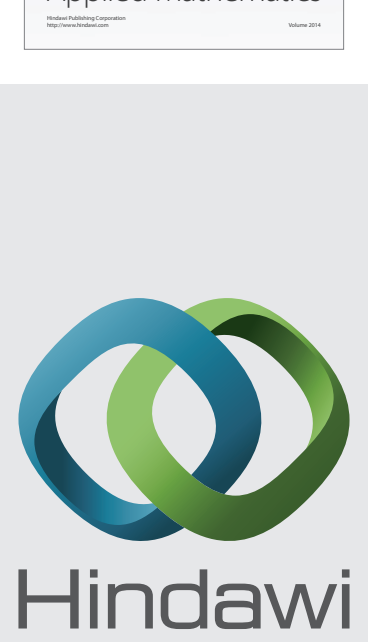

Submit your manuscripts at http://www.hindawi.com
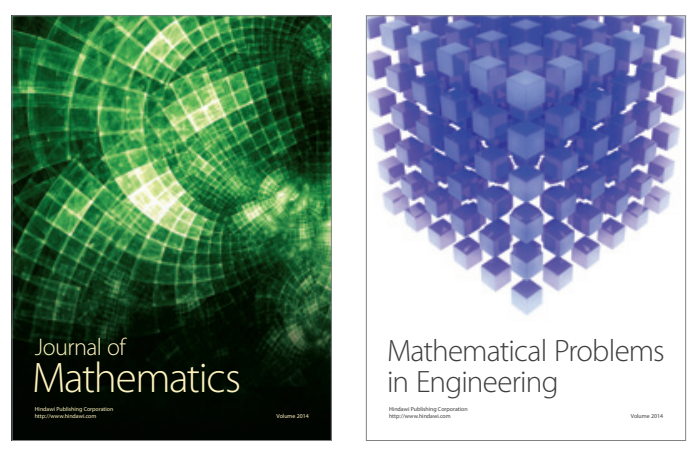

Mathematical Problems in Engineering
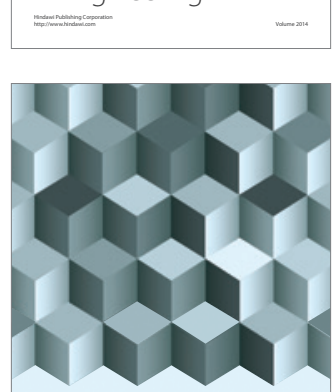

Journal of

Function Spaces
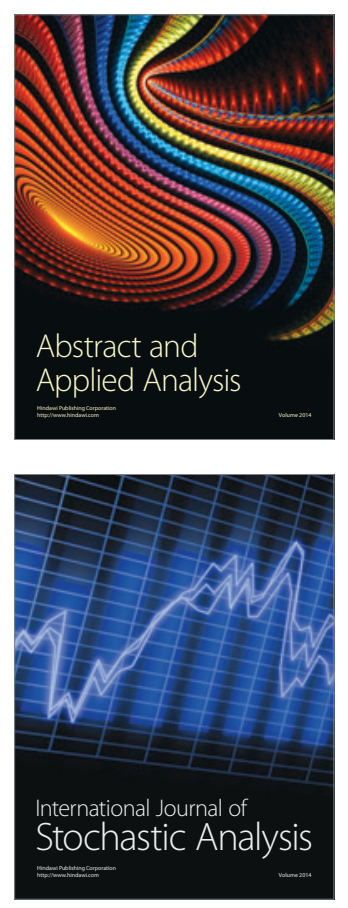

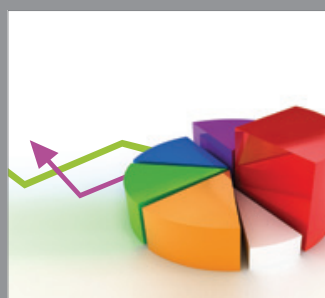

ournal of

Probability and Statistics

Promensencen
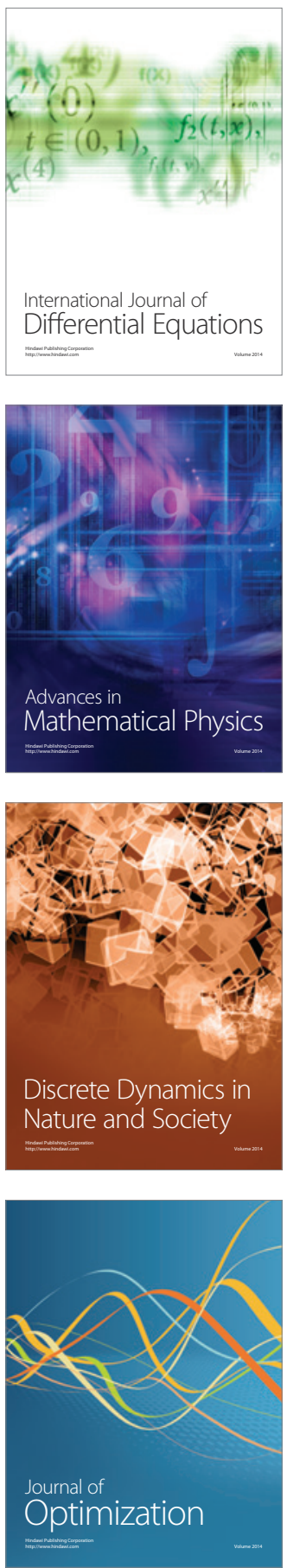